\title{
Search for a Standard Model Higgs boson produced in association with a $\mathbf{W}$ or $\mathrm{Z}$ boson decaying to bottom quarks using the Run 1 data sample in CMS
}

\author{
Stephane Cooperstein ${ }^{* \dagger}$ \\ Princeton University \\ E-mail: stephane.b.cooperstein@cern.ch
}

\begin{abstract}
A search for a Standard Model Higgs boson decaying to $\mathrm{b} \bar{b}$ produced in association with a $\mathrm{W}$ or $\mathrm{Z}$ boson is reported for the following channels: $\mathrm{W}(\mathrm{e} v) \mathrm{H}, \mathrm{W}(\mu v) \mathrm{H}, \mathrm{W}(\tau v) \mathrm{H}, \mathrm{Z}(\mu \mu) \mathrm{H}, \mathrm{Z}(\mathrm{ee}) \mathrm{H}$, and $\mathrm{Z}(v v) \mathrm{H}$. The search is performed on data samples corresponding to integrated luminosities of $5.1 \mathrm{fb}^{-1}$ at $\sqrt{s}=7 \mathrm{TeV}$ and $18.9 \mathrm{fb}^{-1}$ at $\sqrt{s}=8 \mathrm{TeV}$, collected by the CMS experiement at the LHC. An excess of events is observed above the expected background with a local significance of 2.1 standard deviations for a Higgs boson with mass $125 \mathrm{GeV}$ consistent with Standard Model expectations. The signal strength corresponding to this excess, relative to that of the Standard Model Higgs boson, was $0.89 \pm 0.43$.
\end{abstract}

38th International Conference on High Energy Physics 3-10 August 2016

Chicago, USA

${ }^{*}$ Speaker.

${ }^{\dagger}$ On behalf of the CMS collaboration 


\section{Introduction}

The discovery of a new boson in 2012 by the ATLAS and CMS collaborations at the LHC provided an important verification of the Standard Model description of electroweak symmetry breaking. In the Standard Model, the electroweak gauge bosons acquire mass via the Brout-EnglertHiggs electroweak symmetry breaking mechanism [1,2]. Quarks and leptons acquire mass via the coupling of the Higgs to fermions through a Yukawa interaction. In this description, the coupling strength of the Higgs boson to fermions is proportional to the fermion mass. Since its original discovery, precision measurements have been made of the Higgs boson mass, spin-parity quantum numbers, and couplings to $\mathrm{W}$ and $\mathrm{Z}$ bosons. The original discovery and the subsequent measurements have relied primarily on the reconstruction of Higgs boson decays to WW, ZZ, and $\gamma \gamma$ boson pairs. ATLAS and CMS have presented the first evidence of the Higgs boson decaying to fermions via reconstruction of $\tau^{+} \tau^{-}$decays [3, 4]. The Standard Model prediction for the Higgs couplings to the top quark has also been indirectly validated through the observed agreement of the gluon-gluon fusion production cross section with the theory prediction. However, evidence for the Higgs boson coupling to down-type quarks remains to be observed. In theories beyond the Standard Model, the masses of the down-type quarks can be acquired through alternative mechanisms. The observation of and measurement of the Higgs boson coupling to down-type fermions is therefore an essential validation of the Standard Model.

For a Standard Model Higgs boson with mass $125 \mathrm{GeV}$, the predicted branching fraction to bottom quarks is approximately $58 \%$. Although the branching fraction for $b \bar{b}$ decays is much larger

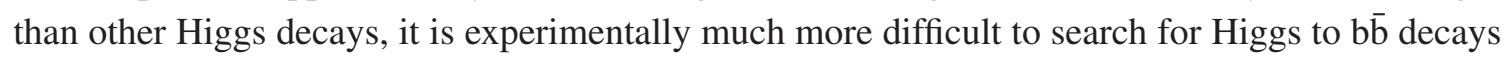
due to the very large QCD multi-jet background.

\section{VH(bb) Topology}

In order to supress the otherwise overwhelming QCD multi-jet background, this analysis reconstructs Higgs boson candidate events produced in association with a vector boson. This production mode is the most sensitive for $h \rightarrow b \bar{b}$ searches at the LHC. The topologies considered are $\mathrm{W}(\mathrm{e} v) \mathrm{H}, \mathrm{W}(\mu v) \mathrm{H}, \mathrm{W}(\tau v) \mathrm{H}, \mathrm{Z}(\mu \mu) \mathrm{H}, \mathrm{Z}(\mathrm{ee}) \mathrm{H}$, and $\mathrm{Z}(v v) \mathrm{H}$. Requiring the presence of the vector boson greatly improves the signal to background ratio, while also providing an efficient trigger strategy. Furthermore, events are required to have a high-momentum pair of b-jets recoiling from the vector boson. This "boost" requirement has the advantage of reducing the relative contribution from vector boson plus jet(s) production, improving the mass resolution, and making the $\mathrm{Z}(v v) \mathrm{H}$ channel accessible through a large missing transverse momentum requirement.

\section{B-jet Energy Regression}

The mass resolution for the reconstructed Higgs candidate is approximately $10 \%$ with a small bias on the mass of a few percent. In order to improve the mass resolution and the bias, a specialized Boosted Decision Tree (BDT) regression is trained on simulated $h \rightarrow b \bar{b}$ events targeting the generator-level $p_{T}$ of the selected b-jets. The resulting improvement in the mass resolution is approximately $15-20 \%$ for the $\mathrm{Z}(11) \mathrm{H}$ modes and $7-12 \%$ for the other modes. The regression 
performance is improved in the $\mathrm{Z}(11) \mathrm{H}$ channel by including the missing transverse energy in the regression training, since in this channel there are no neutrinos from the vector boson decay. The overall improvement on the analysis sensitivity due to the jet energy regression is approximately $15 \%$.

\section{Signal Extraction}

A BDT classifier is trained independently for each sub-channel on simulation to extract signal events from a pre-selected signal region. The set of BDT training input variables is common among channels. The most discriminating variables are the mass of the reconstructed Higgs candidate, the number of additional jets, and the transverse momentum of the vector boson. The output of the BDT classifier is fit to a combination of signal and background templates via a binned maximumlikelihood fit to extract the signal. A re-binning technique is applied to ensure sufficient background Monte Carlo events in each bin.

\section{Systematic Uncertainties}

The normalizations of the primary backgrounds are extracted via simulataneous binned maximumlikelihood fits on the jet b-tagging discriminator output in control regions targeting individually the primary background components in each sub-channel. The fitted normalization scale factors for each background are used as priors in final signal extraction fit with uncertainties ranging from $5 \%$ to $35 \%$. Uncertainties on the diboson and single top expected yields of $15 \%$ are also included, consistent with previous CMS measurements [5, 6].

Data/MC b-tagging scale factors are derived in a heavy flavor enriched control region from jets containing muons and applied to simulation for selected $b$-jets. These scale factors significantly improve the level of agreement between data and simulation for the jet b-tagging discriminator output. The corresponding yield uncertainty is $3-15 \%$ depending on the channel and the specific process. The jet energy scale and jet energy resolution are varied separately one standard deviation, corresponding to yield variations of 2-3\% and 3-6\%, respectively. For each of these sources of experimental systematic uncertainty, both the shape and normalization effects are propogated to the final fits.

The total $\mathrm{VH}(\mathrm{bb})$ production cross section has been calculated at NNLO order accuracy with a corresponding uncertainty of $4 \%$. Because this analysis is performed in a boosted regime, differences between the simulated $\mathrm{V}$ and $\mathrm{H}$ transverse momentum spectra and data can introduce systematic effects on the expected signal acceptance and efficiency. NLO electroweak and NNLO QCD corrections are applied to signal simulation with an uncertainty of $2 \%(5 \%)$ for the electroweak (QCD) corrections.

A small portion of the accepted signal is expected to be from $\mathrm{gg} \rightarrow \mathrm{ZH}(\mathrm{bb})$. This was considered as a separate signal contribution later in the Higgs combination result $[9,10]$.

\section{Results}

A VH(bb) signal is extracted via a simultaneous binned maximum-likelihood fit of the BDT classifier score in each sub-channel. An excess of events above the expected background is ob- 
served with a significance of 2.1 standard deviations. The signal strength of this excess, relative to a Standard Model Higgs boson with mass $125 \mathrm{GeV}$, is $\mu=0.89 \pm 0.43$ Figure 1 (left plot) shows the observed data in the signal region in bins of expected signal to background ratio combined in all channels.

\section{Diboson Extraction}

Standard Model VZ(bb) events have very similar topology to the $\mathrm{VH}(\mathrm{bb})$ signal with a significantly larger cross production cross section. A separate BDT classifier is trained to extract a $\mathrm{VZ}(\mathrm{bb})$ signal from a combination of background and $\mathrm{VH}(\mathrm{bb})$ signal. Figure 1 (right plot) shows the combined $\mathrm{M}_{\mathrm{bb}}$ distribution with all backgrounds except $\mathrm{VZ}(\mathrm{bb})$ subtracted, with the contribution from each sub-channel weighted by the expected $S /(S+B)$. An excess of events above the expected background is observed with a significance greater than 7 standard deviations. The signal strength of this excess is $\mu_{V V}=1.19_{-0.23}^{+0.28}$.
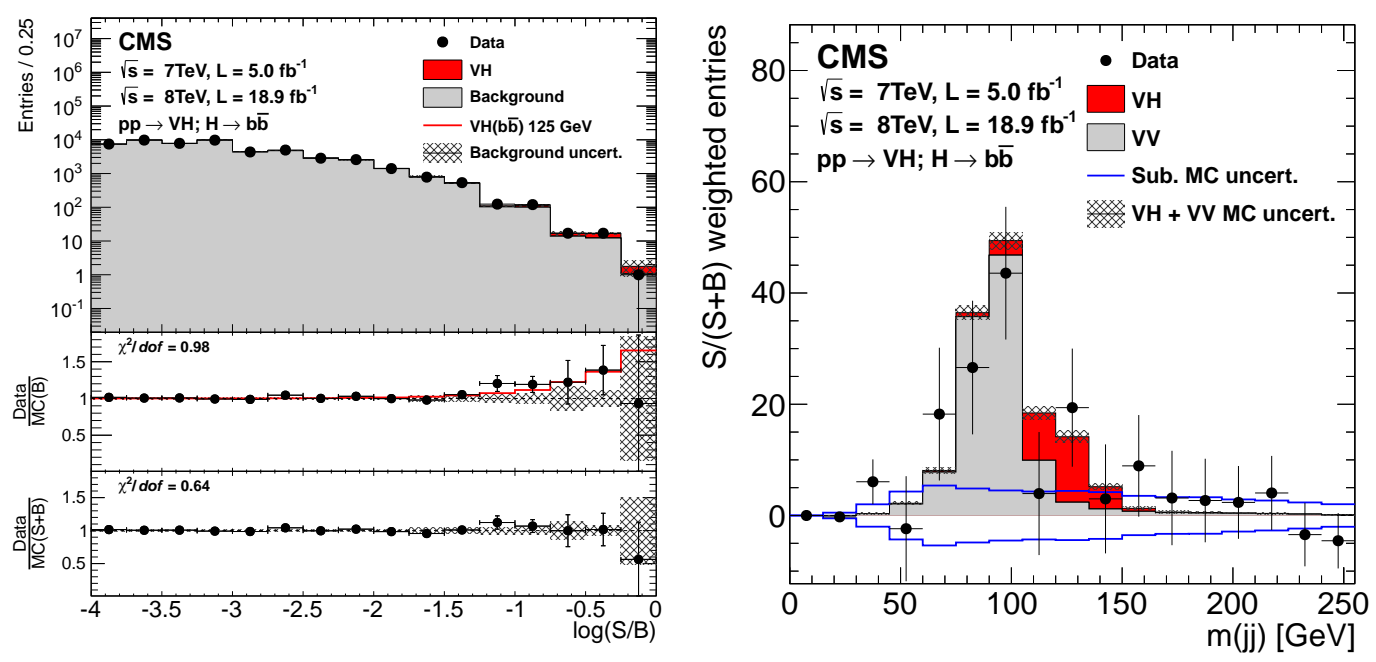

Figure 1: Left: Combination of all $\mathrm{VH}(\mathrm{bb})$ channels into a single distribution. Events are sorted in bins of similar expected signal-to-background ratio, as given by the value of the output of their corresponding BDT discriminant. The bottom two insets show the ratio of data to the background-only prediction (above) and to the predicted sum of background and SM VH(bb) signal (below). Right: S/(S+B) Weighted di-jet invariant mass distribution, combined for all channels with all backgrounds except VV subtracted.

\section{References}

[1] F. Englert and R. Brout, "Broken Symmetry and the Mass of Gauge Vector Mesons," Phys. Rev. Lett. 13 (1964) 321. doi:10.1103/PhysRevLett.13.321

[2] P. W. Higgs, "Broken Symmetries and the Masses of Gauge Bosons," Phys. Rev. Lett. 13 (1964) 508. doi:10.1103/PhysRevLett.13.508

[3] S. Chatrchyan et al. [CMS Collaboration], "Evidence for the $125 \mathrm{GeV}$ Higgs boson decaying to a pair of $\tau$ leptons,” JHEP 1405 (2014) 104 doi:10.1007/JHEP05(2014)104 [arXiv:1401.5041 [hep-ex]]. 
[4] G. Aad et al. [ATLAS Collaboration], "Evidence for the Higgs-boson Yukawa coupling to tau leptons with the ATLAS detector," JHEP 1504 (2015) 117 doi:10.1007/JHEP04(2015)117 [arXiv:1501.04943 [hep-ex]].

[5] CMS Collaboration [CMS Collaboration], "Measurement of the single-top t-channel cross section in pp collisions at centre-of-mass energy of $8 \mathrm{TeV}$," CMS-PAS-TOP-12-011.

[6] S. Chatrchyan et al. [CMS Collaboration], "Measurement of $\mathrm{W}^{+} \mathrm{W}^{-}$and $\mathrm{ZZ}$ production cross sections in pp collisions at sqrt(s) = 8 TeV,” Phys. Lett. B 721 (2013) 190 doi:10.1016/j.physletb.2013.03.027 [arXiv:1301.4698 [hep-ex]].

[7] S. Chatrchyan et al. [CMS Collaboration], "Search for the standard model Higgs boson produced in association with a W or a Z boson and decaying to bottom quarks," Phys. Rev. D 89 (2014) no.1, 012003 doi:10.1103/PhysRevD.89.012003 [arXiv:1310.3687 [hep-ex]].

[8] G. Rauco [CMS Collaboration], "Search for the Standard Model Higgs boson produced by vector boson fusion and decaying to bottom quarks," arXiv:1512.08625 [hep-ex].

[9] V. Khachatryan et al. [CMS Collaboration], "Precise determination of the mass of the Higgs boson and tests of compatibility of its couplings with the standard model predictions using proton collisions at 7 and 8 TeV," Eur. Phys. J. C 75 (2015) no.5, 212 doi:10.1140/epjc/s10052-015-3351-7 [arXiv:1412.8662 [hep-ex]].

[10] G. Aad et al. [ATLAS and CMS Collaborations], "Measurements of the Higgs boson production and decay rates and constraints on its couplings from a combined ATLAS and CMS analysis of the LHC pp collision data at $\sqrt{s}=7$ and 8 TeV," JHEP 1608 (2016) 045 doi:10.1007/JHEP08(2016)045 [arXiv:1606.02266 [hep-ex]]. 